RESEARCH HIGHLIGHTS

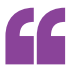

We altered the natural course of the disease in terms of cognitive decline

\title{
Gene therapy for mucopolysaccharidosis shows promise
}

A novel intracerebral gene therapy approach has produced encouraging results in a phase I/II trial in children with mucopolysaccharidosis type IIIB syndrome (MPS IIIB), a lysosomal storage disorder characterized by onset of progressive cognitive deterioration after 2-4 years of seemingly normal development. The findings indicate that the treatment leads to slowing of cognitive decline, especially if administered early in the disease course.

MPS IIIB is caused by mutations in NAGLU, which encodes a- $N$-acetylglucosaminidase. Reduced function of this enzyme results in accumulation of partially degraded heparan sulfate in the lysosomes. The new gene therapy approach was designed to restore the production of functional NAGLU protein in the brain.

"This has been a very long journey, initiated 20 years ago by
Jean-Michel Heard at the Pasteur Institute," explains Marc Tardieu, the lead author of the study. "Preclinical work in MPS IIIB mice and dogs demonstrated that intracerebral injection of a therapeutic vector was highly effective, but only if performed early in the life of the animal."

A recombinant adeno-associated virus $2 / 5$ vector containing the human NAGLU gene was administered intracerebrally to four children with MPS IIIB, aged 20, 26, 30 and 53 months. The children also received immunosuppressive therapy to prevent induction of a deleterious immune response against the therapeutic enzyme.

The study did not include a control group, so the researchers used data from previous studies of the natural history of MPS IIIB to define the expected level of cognitive functioning. All four of the treated children showed better-than-expected cognitive development over a 30-month follow-up period, with the youngest participant showing the best outcome. Despite some adverse events, the treatment was judged to have a favourable safety profile.

"We altered the natural course of the disease in terms of cognitive decline, most probably with a window of opportunity for the best therapeutic efficacy before 2 years of age," concludes Tardieu. "The next step is a new multicentric protocol with a larger number of young patients and delivery of the therapeutic vector both within and outside the brain."

Heather Wood

ORIGINAL ARTICLE Tardieu, M. et al.

Intracerebral gene therapy in children with mucopolysaccharidosis type IIIB syndrome: an uncontrolled phase $1 / 2$ clinical trial. Lancet Neurol. http://dx.doi.org/10.1016/S1474-4422(17)30169-2 (2017) 